Meta

Journal des traducteurs

Translators' Journal

\title{
Pourquoi la porte ne tourne-t-elle pas sur ses gonds?
}

Esquisse d'un modèle explicatif de la cooccurrence par

l'analyse de quatre classes de verbes de déplacement

\section{Massiva N. Zafio}

Volume 40, numéro 1, mars 1995

URI : https://id.erudit.org/iderudit/004634ar

DOI : https://doi.org/10.7202/004634ar

Aller au sommaire du numéro

Éditeur(s)

Les Presses de l'Université de Montréal

ISSN

0026-0452 (imprimé)

1492-1421 (numérique)

Découvrir la revue

Citer cet article

Zafio, M. N. (1995). Pourquoi la porte ne tourne-t-elle pas sur ses gonds? Esquisse d'un modèle explicatif de la cooccurrence par l'analyse de quatre classes de verbes de déplacement. Meta, 40(1), 91-98.

https://doi.org/10.7202/004634ar d'utilisation que vous pouvez consulter en ligne. 


\title{
ÉTUDES TERMINOLOGIQUES ET LINGUISTIQUES
}

\author{
POURQUOI LA PORTE NE TOURNE-T-ELLE PAS SUR SES GONDS? \\ Esquisse d'un modele expllcatif de la cooccurrence par l'analyse \\ de quatre classes de verbes de déplacement
}

Pourquoi la porte ne tourne-1-elle pas sur ses gonds et l'étoile n'oscille-t-elle pas sur son axe? Pourquoi une inflation est dite galopante plutôt que trépidante? Questions étranges de prime abord mais qui voilent un problème incontoumable en langue de spécialité : celui de la cooccurrence. Problème incontournable parce que central (Lethuillier 1991) et d'autant plus intéressant qu'il se prête difficilement à la description. Comment, en effet, concevoir un modèle explicatif qui rende compte d'un phénomène aussi polymorphe (Maingueneau 1991) dans la mesure où il peut sembler résider d'une part dans le contexte situationnel (cas de la porte et de l'étoile). donc extra-linguistique el apparemment syntaxique (cas de l'inflation), d'autre part? L'adjectif galopante paraît le seul apte à modifier le SN sujet inflation dans l'expression de l'intensité (fonction Magn dans le modèle SensTexte de Mel'Cuk); le verbe oscille'r ne semble pas être admis pour exprimer le procès de rotation que décrivent en réalité certaines éloiles. S'agit-il, dans tous ces cas, simplement de bonnes associations entre termes liés par des contraintes pragmatiques, ou existe-t-il, au-delà de la simple combinatoire syntagmatique, des règles d'exclusion de nature autre qui rendent certaines associations acceptables et en rejellent d'autres? question.

Une précision termonologique s'impose avant de tenter de trouver réponse à celle

Cooccurrence et collocation. Les deux notions, en dépit de leur morphologie. renvoient à des réalités distinctes. La collocation est la distribution d'éléments lexicaux qui, partageant des sèmes communs, $n$ 'entretiennent cependant aucune relation grammaticale entre eux. Ainsi, construction et construire sont de même base, appartiennent à des classèmes différents, mais se rencontrent avec les mêmes mots (maison, édifice, etc.), dans le même contextel. Nous ne donnerons de la notion de cooccurrenc' qu une définition minimale, volontairement non satisfaisante, puisqu'elle constitue le propos du présent texte. Étant donné un élément linguistique $\mathrm{x}$, seront ses cooccurrents tous les éléments qui cooccurrent avec lui. Dans la phrase Le petit arbre d'entrainement houge, le déterminant le, l'adjectif petit, le complément de nom d'entrainement et le verbe bouge sont, tous, des cooccurrents de l'élément lexical arbre puisqu'ils apparaissent autour de lui, les uns à sa gauche, et les autres à sa droite.

La notion de cooccurrence ainsi définie n'est que configurationnelle. Elle n'est pas apte à discriminer entre les deux énoncés suivants :

L'arbre d'entrainement tourne à $2(0)(\mathrm{tr} / \mathrm{min}$.

?L'arbre d'entrainement va d $2(x)$ ir $/ \mathrm{min}$.

Elle n'est cependant pas assimilable à celle de bonne association entre termes. Cette dernière, qu'il convient d'expliciter afin de la mieux évacuer, cache deux réalités bien distinctes: la grammaticalité et la recevabilité. L'exemple suivant suffit à élucider les deux concepts :

(1) *Larbre $2(x \times)$ tr / min tourme à.

(2) ? L'arbre va à $2000 \mathrm{tr} / \mathrm{min}$. 
Le premier énoncé est agrammatical pour des raisons évidentes de structure syntaxique. Le second est grammatical mais non recevable en langue technique (tolérable en langue usuelle relâchée).

La non-recevabilité, qui, a contrario, permet de définir la cooccurrence, est-elle le fait de particularités d'énonciation, du poids des contraintes syntaxiques ou des motivations d'ordre sémantique? Là est la question sous-tendant le problème de la cooccurrence au sens où elle est entendue en langue de spécialité.

Il a été, jusqu ici, très ardu de trouver réponse à cette question. une des difficultés étant que l'on ait tenté de rendre compte de tout l'ensemble de ce phénomène polymorphe. Dans le présent texte, nous posons, comme hypothèse, que la cooccurrencé est un phénomène sémantique régi par le verhe. Chaque verbe ou, plus commodément, chaque classe de verbes. comporte un schéma de sens qui met en relation deux ou plusieurs variables (les actants). La bonne cooccurrence est le résultat de l'adéquation entre ce schéma de sens et la relation qu'il établit avec les actants du verhe ( $X$ et $Y$ ).

\section{Verbe}

$\mathrm{X}$
$========>$ PROCÈS

Y

Afin de démontrer cette hypothèse, nous distinguerons deux niveaux d'analyse : le niveau phrastique où nous appliquerons l'analyse aux énoncés de structure SVO. et le niveau infra-phrasique qui étudiera les énoncés de structure moins élaborée (SP et autres).

Il existe, pour décrire la phrase, de nombreux modèles. Certains privilégient le terme sujet (Lethuillier 1991), d'autres le verbe (la grammaire générative par la sous-calégorisation). Nous centrons le nôtre sur le prédicat verbal. L'analyse des verbes de mouvement ou. plus spécifiquement. de déplacement servira de mode de preuve. Nous allons les traiter non pas isolément mais en classes, traitement qui autorise une saisie plus commode de leur sens de base (central meaning) (Porzig 1934. cité par Lyons 1977). Ce sens, une fois circonscrit, sera schématisé.

Les verbes de déplacement, en langue usuelle, ont souvent été étudiés. Nous ne citerons que deux études: celle de Boons (1985), dans une perspective classificatoire et de formalisation, et celle de Lamiroy (198.3), entreprise selon une optique à la fois comparatiste (français / espagnol) et sémantico-syntaxique (verbess de mouvement et constructions infinitives). De ces verbes. Boons propose une définition générale fort satisfaisante: un verbe est dit de mouvement ou de déplacement quand il implique un achangement obligatoire de lieu d'un corps ne subissant par ailleurs aucune modification de forme ni de substance au cours du procès».

Notre étude prend pour cadre le domaine de la mécanique où on peut identifier quatre types de verbes de déplacement:

a) verbes de déplacement linéaire ou translatifs. Ils expriment le passage d un mobile $X$ d'un point $A$ à un point $B, B$ ćtant différent de $A$ ou indéfini :

b) verbes de déplaceme'nt angulaire décrivant un procès se confondant avec une course de $\mathrm{X}$ selon un are de cercle dont le centre sert de pivot:

c) verbes de rotation exprimant un déplacement de $X$ autour de son propre axe:

d) verbes de déplacement quekenquee. ainsi qualitiés du fait que le procès exprimé s'effectuait selon une directionnalité non définie. 


\section{Ces verbes sont:}

classe a : avancer, reculer, entraîner :

classe b: pivoter, sarticuler sur, osciller:

classe c: tourner, entraîner :

classe d: bouger, jouer, se déplacer.

L'analyse du sens des verbes ne peut être envisagée sans que soient posées au préalable la question du critère (Boons 1987) et celle des conditions pertinentes (Lyons 1977 : 26.5). Le premier critère qui nous semble opératoire est celui du procès de déplacement avec ses deux bornes $A$ et $B$, l'une étant la source. l'autre le but. Le second critère conceme la nature du mouvement, c'est-à-dire son statut intrinsèque (automotion) ou extrinsèque (asservissement). Le troisième critère, qui ne jouera que pour l'analyse de certains verbes, est le point d'application de la force engendrant le mouvement décrit par le verbe.

La présence du premier critère et d'un élément des autres critères constitue la condition suffisante fondant le statut de verbe de déplacement. Nous recourrons a l'un ou à l'autre de ces critères au besoin.

\section{LES VERBES DE DEPLACEMENT LINEAIRE OU VERBES TRANSI.ATIFS} but $\mathbf{B}$.

La translation est le déplacement d'un mobile $X \mathrm{~d}$ 'un point source $A$ vers un point

(3) L'outil de coupe avance par intermittence.

Ici. le corrélat de lieu (Boons), le corrélat de déplacement. c'est-à-dire «la chose située par rapport au lieu", ou mieux la chose qui change de lieu. est l'outil de coupe X. Si l'on prend un point théorique $A$ comme source, nous dirons qu'il y a procès d'éloignement de $X$ de $A$ vers un certain point B. B n'est cependant pas nécessaire au procès. Alancer, en langue usuelle, peut avoir deux directionnalités ou aucune, en l'absence d'indications pragmatiques.

(4) Avance-toi près de moi !

Cet énoncé peut être interprété comme un ordre donné à $X$ de s'approcher du "centre déictique, conjonction des trois entités : moi, ici, maintenant» (Bouchard 1992). Le procès décrit est une relation de rapprochement, sens que confirme le Petir Robert qui donne le verbe avancer comme synonyme d approcher.

(5) Avance-1oi!

est ambiguë comme phrase. Certains éléments situationnels sont nécessaires pour en désambiguïser le sens.

(6) Avance!

peut s'analyser comme en (5). L'emploi technique est l'inverse de l'emploi courant. Ce sémantisme est confirmé par le Lexis dans la définition de la notion d'avance :

Déplacement de l'outil d'une machine, après chaque course pour l'amener devant une nouvelle partie de la piece à usiner.

Avancer, au sens mécanique, implique une relation d'éloignement intrinsèque que véhicule le SP “pour l'amener devant une nouvelle partie de la pièce à usiner». Éloignement par rapport à quoi?, serait-on en droit de se demander. Il nous faut ici introduire un élément 
du second critère mentionné plus haut: les concepts d'état initial et d'état final (Boons 1985). Si l'on admet que A est assimilable à l'état initial. l'outil serait dans cet état à sa mise en marche. Chaque course correspond donc à un éloignement par rapport à ce point.

Résumons: arancer, dans son acception technique, exprime une relation d'éloignement, sens contraire au sémantisme du même prédicat dans sès emplois usuels.

\section{Reculer}

Il porte le même sens technique qu'arancer. Ici comme là nous avons un procès d'éloignement de $X$ d'un point source $A$ vers un but $B$ non explicite.

(8) La planche recule sous l'action de la scie.

(9) L'auto recule.

(10) Il recula de deux pas.

Le déplacement admet comme point d'application le dos de X $(9$ et 10 ). Pour les objets "sans orientation spécifique" (Vandeloise 1986), ce point n'est pas défini. Tous ces emplois demeurent néanmoins en congruence avec le sens courant.

\section{Entraîner}

Entrainer est un verbe translatif intéressant à plus d'un titre. Il met nécessairement en relation deux actants, ce qui obscurcit quelque peu le statut intrinsèque ou extrinsèque du procès.

(11) La locomotive entraîne les wagons.

À quel item assigner $X$, d'autant que les deux actants sont mobiles? Pour la commodité de l'analyse, nous sommes obligé de distinguer deux variables $X$ et $Y$. La première variable $(X)$ serait plus agentive que la seconde $(Y)$. Nous appliquons notre analyse à $X$. qui sera la locomotive. Elant entendu que $X$ est menant (driving). $Y$ ne pourra être que mené (driven). Son mouvement est asservi à celui de X. La relation expriméce est une relation de traction. Et l'on sait que dans un proces de traction, $X$ est devant $Y$, les deux peuvent être ou non contigus (lexpression traction arriere est. à cause de cela, une aberration). Nous demeurons toujours dans la translation. Le mouvement est intrinsèque du point de vue de $X$, et extrinsèque par rapport à $Y$.

Il ressort de cette analyse des verbes de déplacement linéaire certaines constantes (parcours linéaire du mobile, nécessité de la source mais pas du but) et. aussi. certaines divergences. Avancer. dans son emploi technique, porte un sens inverse du sens usuel et a le sens de reculer puisqu il exprime une relation d'éloignement. Entraine'r a une structure actantielle différente et établit une relation assez complexe entre deux actants. Son analyse nécessite l'introduction de la notion de point d'application, essentielle au procès de traction.

Il est possible d'envisager tous ces sens de façon unifié afin d'isoler leur senss de' basé. Ils correspondent à un schéma général commun (le schéma de sens), admettent une source $A$ et un mobile ( $X$ ou $Y$ ) et expriment une relation d'éloignement. Ce schéma serait le suivant:

\begin{tabular}{ll} 
& \multicolumn{1}{c}{$\mathrm{X}$} \\
où $\quad$ est la source. \\
$\mathrm{A}$ & lé mobile. \\
& l'éloignement et forme du parcours. \\
& le but.
\end{tabular}


Ce schéma rend compte du sens des trois verbes de la classe A. Et la question de la cooccurrence? Elle n'est pas laissée pour compte. Son traitement sera plus aisé une fois établi que la recevabilité esı conditionnée par le sens du verbe. Aussi, poursuivons l'analyse.

\section{CI.ASSE B : I.ES VERBES DE. DEPLACEMFNT ANG'UI.AIRF.}

Le déplacement angulaire est la course d'un mobile $\mathrm{X}$ selon un arc de cercle dont le centre sert de pivot. Dans cette classe, nous avons retenu trois verbes: pivoter, osciller et s"articuler.

\section{Pivoter}

(12) La chape pivote sur son axe.

(13) La porte pivote sur ses gonds.

(14) "ll pivota sur ses talons et se retrouva devant son fourneau." (Lexis)

Dans le dernier énoncé, le parcours s'effectue néanmoins selon un arc de cercle. même si le Lexis donne ce verbe comme synonyme de tourner.

(15) ?La porte tourne sur ses gonds.

À moins qu'il ne s’agisse d'une porte à tourniquet, l'énoncé (15) n'est pas recevable. De façon prototypique, c'est-à-dire courante, une porte ne peut tourner sur ses gonds, la structure du cadre l'en empêchant ( «La lourde porte tourna sur ses gonds." (Balzac) nous semble relever de la licence littéraire).

\section{S'articuler sur}

Ce verbe porte le même sens que le prédicat précédent avec lequel il entretient un étroit rapport de sens (Porzig 1934 cité par Lyons 1977), une relation de synonymie. Le Lexis donne la définition suivante du mot articulation:

En mécanique, assemblage dont l'élément de liaison est constitué par un axe permettant un déplacement angulaire des pièces assemblées.

Cette définition, transférée au verbe. confirme la relation de synonymie entre les lexèmes s'articuler et pivoter.

\section{Osciller}

Osciller est un verbe de déplacement angulaire qui décrit un procès un peu plus complexe que les deux précédents.

(16) Le tableau oscille sur le mur après le tremblement de terre.

(17) Le pendule oscille.

Le déplacement décrit s'effectue selon un arc de cercle dans un mouvement d'aller-retour de $X$ par rapport à un point de référence considéré comme pivot. A source et $B$ but s'alternent. Dans osciller entendu au sens non mécanique (électronique, par exemple), le mouvement altematif est associé à un déplacement linéaire. Le procès se déroule dans un plan horizontal.

(18) L'onde acoustique oscille.

Le verbe qui, en mécanique, véhiculerait ce sens est rehondir.

(19) La balle rebondit sur le plancher.

(20) La balle rebondit sur le mur. 
Dans l'énoncé (19), la répétition du mouvement est due à la gravité. En (20), le changement de plan impose que la balle soit renvoyée de force pour obtenir la répétition du mouvement.

\section{Représentation schématique}

Pivoter, s'articuler sur, osciller (sens mécanique), présentent des traits communs:

un mobile $\mathrm{X}$ effectue un parcours selon un arc de cercle dans un plan vertical.

Ils different en ce que osciller implique un mouvement alternatif. Nous pouvons schématiser ainsi ces sens :

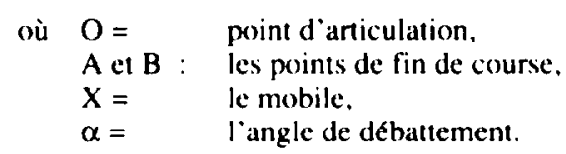

\section{CLASSE C : LES VERBES DE ROTATION OU VERBES GIRATOIRES}

Les verbes de rotation se définissent comme des verbes exprimant le déplacement de $\mathrm{X}$ autour de son propre axe. Peu de verbes en mécanique sont aptes à assumer ce sens.

(21) L'arbre toume a $2000 \mathrm{tr} / \mathrm{min}$.

(22) ?L'arbre avance d $2000 \mathrm{tr} / \mathrm{min}$.

(23) ? L'arbre va à $2000 \mathrm{tr} / \mathrm{min}$.

(24) ?L'arbre roule à $2000 \mathrm{tr} / \mathrm{min}$.

(25) ?La porte tourne sur ses gonds.

Une schématisation du sens permettra d'analyser la non-recevabilité des énoncés (22) à (25).

où $\mathrm{o}=$ axe de rotation.

$=$ parcours circulaire.

$\mathrm{X}=$ le mobile.

Énoncé (22): avancer, avons-nous dit, exprimait un déplacement en translation, c'esta-dire une trajectoire rectiligne.

Énoncé (23): aller est prototypique comme verbe de déplacement (Bouchard 1992). Il implique le passage d'un mobile $\mathrm{X} d$ 'un point $\mathrm{A}$ vers un centre déictique de localisation variable. C'est, du moins, un des sens le plus courant.

Énoncé (24): rouler : se déplacer en faisant se tourner sur lui-même un mobile X comme dans «il roule un tonneau». Au procès de rotation vient s'ajouter une composante translation, ce qui n'est pas conforme au schéma du sens.

Énoncé (25): tourner n'est pas adéquat car il n'y a pas mouvement giratoire de la porte.

\section{CLASSE D : LES VERBES DE DEPLACEMENT QUELCONQUE:}

Nous définissons ces verbes comme décrivant un procès se déroulant sans directionnalité bien assignée. Ce sont : jouer, bouger, se déplacer.

\section{Jouer}

Ce lexème se définit comme étant le «déplacement intempestif d'une pièce résultant d'un défaut de serrage dû à l'usure" (Le'xis). Cest le sens du verbe dans les énoncés suivants:

(26) La chape joue sur son axe.

(27) La porte joue sur ses gonds. 
Avons-nous affaire au même procès dans ces deux énoncés? Que non. Dans jouer, nous avons un déplacement non désiré, intempestif, résultat d'un defaut mécanique.

(28) La porte bouge librement.

(29) ?La piece joue librement.

Dans bouger, nous avons un déplacement sans spécification de la cause de cette action. Le sens de bouger est plus large que celui de jouer qui, lui, est non seulement restreint mais marqué négativement. Jouer ne peut donc jamais apparaître dans un contexte positif.

\section{Se déplacer}

Se déplacer, passer d'un point à un autre. C'est, du point de vue sémantique, le verbe de déplacement primitif; il donne d'ailleurs son nom à la classe des verbes qui expriment son sens. $X$ se déplace quand il passe d'un point source $A$ vers un but $B$.

Que dire de la directionnalité du procès de déplacement dans ces verbes? Elle est quelconque. Et l'on peut, en poussant plus loin l'analyse, dire qu'ils empruntent leur directionnalité à l'une ou l'autre classe de verbes. Aussi est-il difficile de proposer une représentation schématique de leur sens. Une porte peut jouer sur ses chamières parce qu'on peut la soulever légèrement: il y a un jeu. un espace libre, entre la partie de la chamière solidaire de la porte et celle solidaire du cadre. La même porte peut jouer du fait de l'amincissement de l'axe d'articulation. Nous avons un léger mouvement de translation.

Avant de clore cette analyse sémantique quelque peu fastidieuse, un phénomène intéressant à noter pour qui étudie les verbes de déplacement : la mutabilité ou, autrement dit. la capacité de certains verbes à passer d'une classe à l'autre. Se déplacer peut glisser de la classe des vertes de déplacement quelconque à celle des translatifs. Bouger également. Entrainer, peut passer des translatifs aux verbes giratoires. Ce comportement, selon nous, dépend non de considérations syntagmatiques liées aux fréquences d'occurrence des verbes associés à certains actants mais est justiciable du schéma de sens. Bouger, n'ayant pas de directionnalité définie peut, de ce fait, se conformer au schéma de sens d'une catégorie de verbes avec lesquels il présente certaines compatibilités. Le même raisonnement vaut pour jouer et entrainer.

(31) Le démarreur entraîne le moteur.

(32) La locomotive entraine le wagon.

Dans l'énoncé (31), le procès est rotatif. Le démarreur, parce qu'étant un moteur électrique. entraine le moteur par l'intermédiaire d'engrenages animés de mouvement de rotation. L'ensemble de l'événement se déroule en rotation. ce qui explique que l'on rencontre parfois l'expression entraîner en rotation. L'énoncé (32) décrit une relation translative trop courante pour mériter qu'on s'y attarde.

\section{LE PROBLEME DE LA COOCCURRENCE}

Après ce détour, volontairement long, nous pouvons reposer la question de la cooccurrence, pour les énoncés phrastiques, en des termes plus empiriques : comment expliquer la non-recevabilité de tous les énoncés précédés du point interrogatif?

- ?La porte toume sur ses gonds.

- ?La chape bouge sur ses gonds.

- ?La porte oscille sur ses gonds.

- ?L'arbre avance à 2000 ) tr $/ \mathrm{min}$, etc. 
Cette non-recevabilité ne découle pas du SN sujet mais de la sémantique du verbe. Le sens des verbes correspond à un schéma précis dont nous avons essayé de donner une représentation. Bien plus, la non-recevabilité découle de l'inadéquation entre le schéma sémantique et le rôle que jouent les actants par rapport à ce schéma. Tourner, du fait de son schéma de sens "déplacement de X autour de son propre axe" ne peut admettre à gauche (en position sujet) et à droite (complémentation) des items lexicaux non compatibles avec le schéma. C'est donc ce schéma qui impose, ultimement, des contraintes de sélection (Katz. 1973), réduit et détermine la relation entre les items lexicaux devant apparaître en position sujet et complément. L'énoncé : Av'ance-toi était ininterprétable à cause de la directionnalité. La porte tourne sur ses gonds n'est pas recevable à cause du schéma sémantique du verbe tourner et de l'inadéquation de la relation de rotation que ce verbe établit entre le SN porte et le SP sur ses gonds. Tous ces énoncés sont, au demeurant, grammaticaux. Aussi, dire de tourner qu'il représente le bon cooccurrent verbal de porte est mal poser le problème. Car ce qu'il s'agit d'exprimer primitivement n'est pas la notion de porte. mais l'action que subit cette porte. C'est sur cette action que doit se centrer l'explication de la recevabilité de l'énoncé.

Un tel modèle explicatif peut-il être généralisable au-delà de la mécanique qui, au départ, offre l'avantage de présenter des verbes dont le sens se prête à une description fonctionnelle (Vandeloise 1986) du genre que nous venons d'effectuer? Nous pensons que oui. Tout verbe technique est porteur d'un sens, qu'à défaut de schéma on peut parvenir à modéliser. Si l'hypothèse ontogénétique de Trier (1934) (cité par Lyons 1977) selon laquelle tous les lexèmes ont primitivement porté un sens concret avant de dénoter un champ lexical abstrait est vraie, il est permis de croire qu'une analyse fine et rigoureuse des classes de verbes dans un domaine relativement comme l'électricité permettrait la mise au jour de tels schémas.

Que dire de la cooccurrence adjectivale, prépositionnelle, adverbiale? Ces éléments constituent des éléments que nous avions appelés infra-phrastiques. On ne peut donc leur appliquer le même modèle d'analyse. Néanmoins, ici comme ailleurs, nous pensons que la recevabilité est assujettie à des contraintes sémantiques. La suite inflation galopanté met en jeu un trope, un transfers de sens (Cressot 1967), le transfert métaphorique de l'idée de vitesse d'un animal domesticable vers celle d'une situation hors de contrôle. Autant que le cheval, les soubresauts de l'économie peuvent être contrôlables. En cas de crise, ces entités peuvent afficher des comportements débridés. La prégnance du fait sémantique est évidente. Dans le phrasème grièvement hlessé que nous considérons comme phénomène infra-phrastique car, même inséré dans une phrase du genre : «lsabelle se blessa grièvement en tombant", il est évident que le problème découle de la caractérisation du verbe blesser par l'intensif grièvement. Pourquoi cet intensif plutôt qu'un autre? Emploi arbitraire, dirait Mel'cuk. "C"est sans doute parce que cet adverbe est un archaïsme qui ne s'emploie guère qu'avec blessé"», explique Darbelnet (1988: 134). L'étude de la cooccurrence des éléments infra-phrastiques, à n'en point douter, n'est pas tâche aisée. Les solutions syntaxiques proposées jusque-là sous divers vocables («combinatoire d'usage" Lethuillier 1991 ; «toumures idiomatiques propres à un domaine». Gaulthier 1976: voire la sous-catégorisation et la compositionnalité chez. Marantz. 1988) sont, à des degrés variables, plus constatatives qu'explicatives. Il y aurait lieu de porter la réflexion au-delà de l'argumentation syntaxique. La recevabilité des parties infra-phrastiques du discours ne relèverait-elle pas, elle aussi, de l'adéquation entre le sens de ces constituants et le réseau notionnel (sémantique) dans lequel ils s'inscrivent? Nous nous prêterons à un tel exercice à une autre occasion. Nous essaierons alors de vérifier la généralisation de notre modèle d'analyse par l'étude de ces énoncés et, secondement, dans la même perspective, nous effectuerons l'analyse d'une classe de verbes qui opèrent aussi bien en mécanique qu'en 
électricité : les verbes exprimant la relation de liaison. «Pourquoi la porte ne toume-t-elle pas sur ses gonds et l'étoile n'oscille-t-elle pas sur son axe?» La réponse est dans le verbe.

Massiva N. Zafio

Universite du Québec a Trois-Rivieres, Trois-Rivieres, Canada

\section{Notes}

1. Cette définition de la collocation. proposée par Ducrot et Todorov. est très eloigné de celle qu'en donnent Ménard (1989) et Francine Bergevin (1991). Ces demiers considerent la collocation comme un phénomène régi par des contraintes sémantiques, ce qui justifie sa prise en compte dans la mesure des relations lexicosémantiques, pour l'un, et dans l'étude de la cohésion, pour l'autre.

\section{REFERENCES}

BOONS. Jean-Paul (1985) : «Préliminaires à la classification des verbes locatifs : les compléments de lieu, leurs valeurs aspectuelles $*$. Linguistica Investigationes, IX, $\mathrm{n}^{\circ} 2$, Amsterdam. John Benjamins.

BOONS. Jean-Paul (1987) : «La notion sémantique de déplacement dans une classification syntaxique des verbes locatifsn (photocopie).

BOUCHARD. Denis (à paraitre) : The Semantics of Syntax.

CRESSOT, Marcel (19(6) : Le sfyle et ses techniques. Paris, Hachette.

DARBELNET, Jean (198K) : "L'apport de la stylistique comparte a l'enseignement de la traduction*, Meta, 33-2. pp. 133-141.

DUBOIS ef al (1973): Dictionnaire de linguistique, Paris, Larousse.

LAMIROY. Beatrice ( 1983 ) : Les verbes de mouvement en franşais el en espagnol. Amsterdam et Philadelphic. Presses de l'Université de Louvain.

LETHUILLIER, Jacques (1991): «Combinatoire, terminologies et textes*, Meta, 36-1, pp. 92-100.

LYONS, John (1977) : Semuntics, Cambridge, Cambridge University Press

MAINGENEAU, Dominique (1991): L'analyse du discours. Intreduction anx lectures de l'archive, Paris, Hachette.

MÉNARD, Nathan ( 1989 ): «Mesure des relations lexico-sémantiques dans des textes scientifiques: problemes méthodologiques $m$. Meta, 34-3, pp. 468-478.

VANDELOISE. Claude (1986) : L'espace en français - Semantique des prepositions spasiales. Paris. Editions du Seuil. 